\title{
New Insights into the Assembly and Metabolism of ApoB-Containing Lipoproteins from in vivo Kinetic Studies: Results on Healthy Subjects and Patients with Chronic Kidney Disease
}

\author{
Benjamin Dieplinger and Hans Dieplinger
}

Additional information is available at the end of the chapter

http://dx.doi.org/10.5772/51865

\section{Introduction}

Lipoproteins are complexes consisting of a lipid core of mainly triglycerides and cholesterol esters surrounded by a surface monolayer of phospholipids, free cholesterol and specific protein components named apolipoproteins [1]. Most apolipoproteins undergo complex exchange reactions and serve many metabolic functions including transport, enzyme cofactors and receptor ligands. Except for the covalently linked apolipoprotein(a)apolipoproteinB-100 (apo(a)-apoB) complex in Lipoprotein(a) [Lp(a)], apolipoproteins are non-covalently associated with each other and the lipid core.

Lipoprotein disorders are often associated with cardiovascular disease (CVD), atherosclerosis and other organ dysfunctions $[2,3]$. To prevent and treat these diseases and to fully understand their cause, it is necessary to characterise the underlying metabolic disorders [1]. The conventional initial approach to do this is by measuring concentrations of plasma lipids or apolipoproteins. However, abnormal concentrations of lipids and apolipoproteins can result from changes in the production, conversion or catabolism of lipoprotein particles. Therefore, although static measurements and functional assays are important techniques to gain first in vivo functional insights, it is necessary to study their metabolic pathway to understand the complexity of lipoprotein function and pathophysiology $[4,5]$.

Animal models cannot sufficiently replace human studies to explore lipoprotein metabolism due to substantial species specificity. This holds particularly true for conventional 
laboratory animals such as mice and rats which - unless genetically modified or induced by special diet - do not develop atherosclerosis (see review [6]). The same argument is valid for investigations using cellular model systems. Since the liver is the central organ responsible for lipoprotein metabolism and primary human hepatocytes are of only limited use in research, most cellular studies in lipoprotein metabolism have been conducted in human hepatoma cells lines. These lines express, secrete and assemble a lipoprotein pattern which is substantially different from the respective human counterpart [7].

For all these reasons, the in vivo investigation of metabolic pathways in human subjects is the ultimate approach to elucidate physiological or pathological functions of metabolites in the human body. Historically, such human kinetic studies were performed using radioactive tracers; this methodology is, however, nowadays of only restricted use. Therefore, stableisotope tracer kinetic studies in human subjects with clear advantages regarding safety and technical issues have replaced the radiotracer methods to become an important research tool for achieving a quantitative understanding of the dynamics of metabolic processes in vivo.

The aim of this review is to shortly describe the methodology and illustrate how the approach has expanded our understanding of physiological mechanisms as well as the pathogenesis of disorders of human lipoprotein metabolism. We will then specifically address the assembly mechanism of the atherogenic Lp(a) complex and focus on the kinetics of apoB-containing lipoproteins in patients with chronic kidney disease. This patient group is well-known for its high risk for atherosclerotic complications and a 10- to 20-fold increased cardiovascular mortality compared to the general population [8].

\section{Principles of tracer technology}

Exogenous and endogenous labelling techniques have been used to study the in vivo metabolism of an endogenous molecule, the tracee (see review [4]). In the exogenous method, the same molecule, in form of a usually radioactively labelled tracer, is introduced into the bloodstream [9]. In lipoprotein studies, this methodology first requires purification of the target molecule or particle and ex-vivo radiolabelling followed by reinfusion into the circulation. The physological integrity of the target molecule might, however, suffer from such procedure. Furthermore, in case of multiprotein complexes (which most lipoproteins are), the kinetics of individual protein components cannot be investigated by this approach. As an example, the investigation of in vivo kinetics of both protein components of $L p(a)$, as described in this article, to study its assembly mechanism would not be possible with the exogenous labelling approach.

In contrast, in endogenous labelling, a labelled precursor of the molecule of interest, in case of proteins usually a labelled amino acid, is used to label the target molecule by infusion into the circulation of a suitable proband. Ideally, the tracer can easily be detected and quantified, has the same kinetic behaviour as the tracee, and does not perturb the system. Usually, kinetic studies are performed in steady state, where the rates of input and output for a given unlabelled tracee substance are equal and time invariant. Thus, the information provided by the tracer reflects the behaviour of the tracee [10,11]. At various times, the target protein or particle has to be purified from the blood of human probands and the 
amount of tracer is quantified to provide a kinetic curve. A mathematical model is then constructed to extract all the information contained in the kinetic curve. By fitting a model to the data, it is possible to calculate the parameters of the model that characterize the flux of molecules between kinetically homogeneous pools. For example, it is thus possible to investigate the whole pathway including production, conversion or catabolism of lipoprotein particles, information that cannot be obtained by static measurements alone.

The term stable isotope refers to a non-radioactive isotope of a given atom that is less abundant in a molecule within a biological system than the lightest naturally occurring isotope. The most common stable isotope used as metabolic tracer for apolipoprotein kinetic studies is [2H3]-leucine. Stable isotope tracers are much safer than radioactive tracers for both the study subject and the investigator. Furthermore, the duration of stable isotope experiments is normally less than 24 hours which is much shorter compared to radiotracer techniques which may need up to 14 days of examination [9].

\subsection{Tracer administration}

A tracer can be administered intravenously as either a single bolus injection, a primed constant infusion (i.e., a constant infusion given immediately after a priming bolus), or as a combination of both. The tracer bolus administration offers superior dynamics compared with the primed constant infusion, because the enrichment curves (the tracer/tracee ratios) after a bolus injection correspond to the impulse response of the system. It is therefore suitable to study components of lipoprotein metabolism with a slow rate of turnover. Another advantage of bolus administration is that it facilitates the determination of newly synthesized particles, as the intracellular precursor enrichment is greater at the start of the study. This argument therefore counts particularly when investigating kinetics of particle assembly, as described in 3.1.1. Practically, the bolus infusion is also most convenient for both subjects and investigators.

\subsection{Multicompartment models for data analysis}

Multicompartment modelling is a superior method to dissect the complexities of lipoprotein metabolism, and has been widely applied to systems in which material is transferred over time between compartments connected in a specific structure to permit the movement of material amongst the compartments [12].

Each compartment is assumed to be a homogenous entity within which the entities being modelled are equivalent. For instance, the compartments may represent different types of lipoprotein particles that are kinetically homogeneous and distinct from other material in the system. Very often, the data can be described by more than one model. To ensure that the best model is selected, it is necessary to carefully examine the fitting of the kinetic curve, to determine the precision of the parameter estimates, and to perform statistical tests to compare results obtained with different models. However, the complexity of a multicompartment model is usually a compromise for what is practically possible. A very simple model may not adequately describe the kinetic heterogeneity present within the system. A model that is too complex, on the other hand, will not be supported by 
experimental data and, hence, will have little predictive value. Furthermore, even if the development of models is based on experimental data, several assumptions are required in order to derive the model that is to be used. Thus, mathematical models do not determine the kinetics of lipids directly; rather, they derive an indirect approximation.

The software SAAM (Epsilon Group, Charlottesville, VA, USA) has become the first choice for modelling lipoprotein kinetic studies. The SAAM II program was recently developed by SAAM Inst., Inc., Seattle, WA, USA, and is frequently used to analyse lipoprotein tracer data using compartmental models [13, 14]. The primary kinetic parameter resulting after modelling with SAAM II is the fractional synthesis rate (FSR) which, under steady state conditions, is identical to the fractional catabolic rate (FCR) and has the dimension of pools/day. The reciprocal value of FSR/FCR is called retention time (RT, given in days) and indicates the residence time of the investigated tracee (the target apolipoprotein in our cases) in the circulation. The product of FSR multiplied by the concentration of tracee is called production rate (PR) and is usually expressed as $\mathrm{mg} / \mathrm{kg}$ body weight/day.

\section{Metabolism of apoB-containing lipoproteins}

Dietary lipids are absorbed in the intestine and packaged into large, triglyceride-rich chylomicrons which undergo lipolysis to form chylomicron remnants. In the last step of the so-called exogenous lipoprotein pathway, these particles are finally taken up by the liver. The liver then secretes triglyceride-rich lipoproteins known as very low-density lipoproteins (VLDLs) representing the first step oft the endogenous lipoprotein pathway (Figure 1). Lipoprotein kinetic studies have shown that VLDLs are metabolically heterogeneous. Following lipolysis by endothelium-bound lipoprotein lipase (LPL) and hepatic lipase (HL), these particles are converted via intermediate-density lipoproteins (IDL, also called VLDL remnants) to low-density lipoprotein (LDL) or taken up by the liver. LDL is catabolized mainly by the liver or peripheral tissues via the LDL receptor. Increased plasma concentrations of LDL are a major risk factor for CVD. ApoB-100 is the major apolipoprotein of chylomicrons, VLDL, IDL and LDL.

Lipoprotein(a) [Lp(a)] consists of an LDL-like particle which is covalently bound to the glycoprotein apolipoprotein(a) [apo(a)] by disulfide linkage and derives from the liver [15] (Figure 2). Among individuals, Lp(a) plasma concentrations vary more than 1000-fold, ranging from less than $0.1 \mathrm{mg} / \mathrm{dl}$ to more than $300 \mathrm{mg} / \mathrm{dl}$. Depending on the investigated population and the used genetic approach, it has been shown that between $30 \%$ and $90 \%$ of this variation in plasma concentrations of $L p(a)$ is determined by the apo(a) gene locus, encoding proteins from $<300$ to $>800 \mathrm{kDa}$ [16-18]. Apo(a) size is negatively correlated with Lp(a) concentrations, such that low-molecular-weight (LMW) apo(a) isoforms express on average high Lp(a) plasma concentrations, while high-molecular-weight (HMW) isoforms are usually associated with lower concentrations (reviewed in reference [15]). Elevated plasma concentrations of $\mathrm{Lp}(\mathrm{a})$ have been found associated with an increased risk of developing CVD in many studies which was confirmed by recent large meta-analyses $[19,20]$. In vivo kinetic studies using radio-labeled $\mathrm{Lp}(\mathrm{a})$ indicated that the large differences in $\mathrm{Lp}(\mathrm{a})$ concentrations seen among individuals are determined by synthesis and not degradation $[9,21]$. 


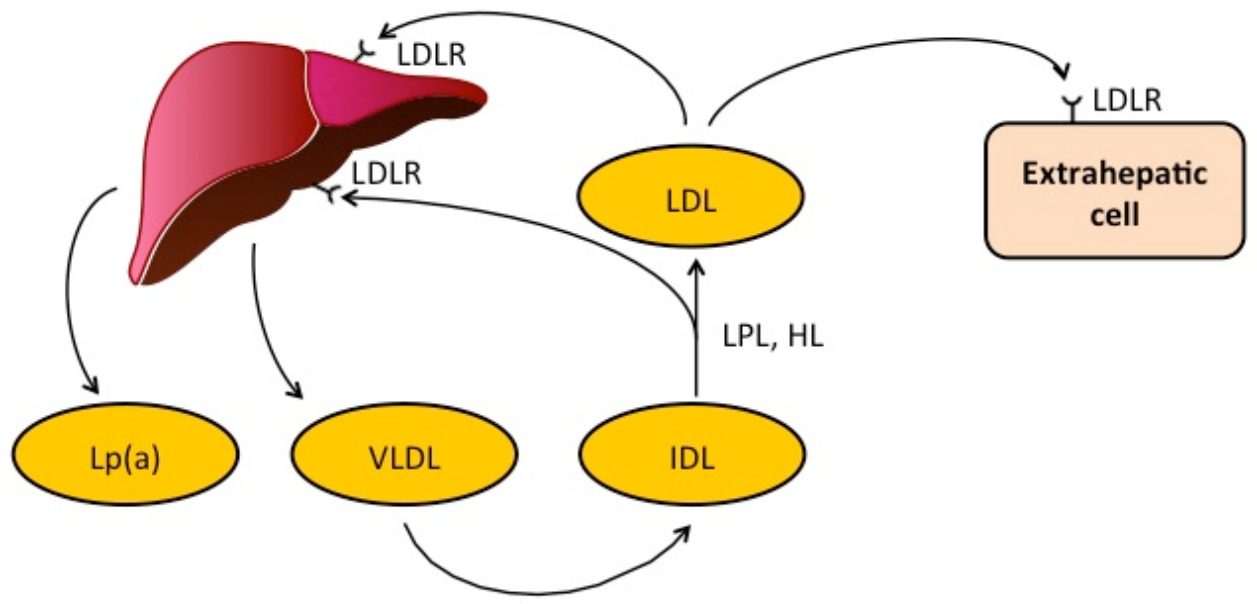

LPL

Figure 1. Endogenous metabolic pathway of apolipoprotein B (apoB)-containing lipoproteins. Triglyceride-rich very-low-density lipoproteins (VLDL) are synthesized and secreted by the liver into the blood stream and their triglycerides catabolized by the endothel-bound enzyme lipoprotein lipase (LPL) resulting in intermediate-densitly lipoproteins (IDL). LPL and hepatic lipase (HL) further convert IDL to low-density lipoproteins (LDL) which are removed from the circulation by the liver and extrahepatic tissue cells via LDL-receptor (LDLR)-mediated endocytosis. Lipoprotein(a) [Lp(a)] is synthesized and secreted by the human liver into circulation.

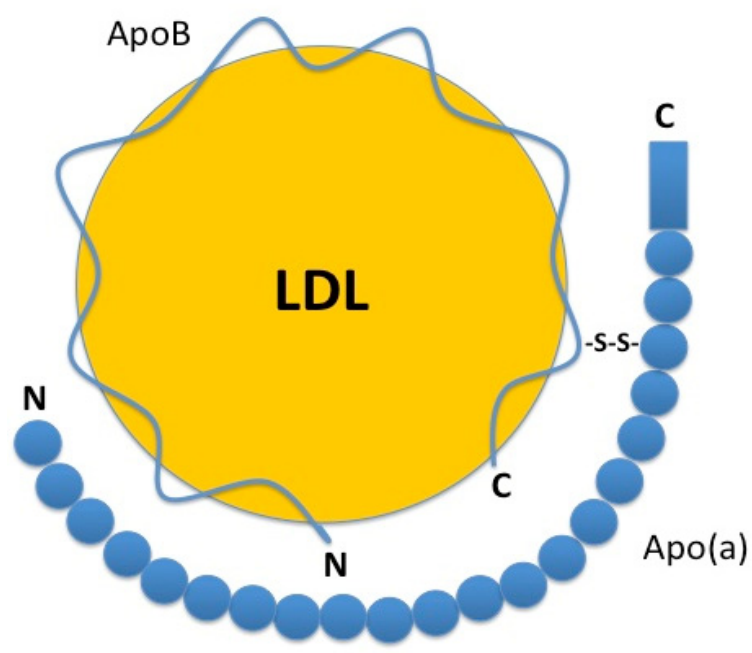

Figure 2. Structure of lipoprotein(a) [Lp(a)]. Lp(a) consists of an LDL-like particle and the disulfidebridge-linked glycoprotein apolipoprotein(a) [apo(a)] which exerts high sequence homology to plasminogen. Apo(a) consists of an inactive protease domain (blue rectangle) and identical as well as non-identical repeats of kringle domains (blue circles). The number of identical kringles vary among individuals and gives rise to a genetically determined molecular size polymorphism of apo(a). 


\subsection{Biosynthesis of $\mathrm{Lp}(\mathrm{a})$}

$\mathrm{Lp}(\mathrm{a})$ has been the target of extensive and successful research particularly with respect to the unusually high degree of genetic control of its expression. In contrast, metabolism and physiological roles as well as pathogenicities of Lp(a) are still poorly understood, as recently reviewed by Dubé et al. [22]. The mechanisms that control Lp(a) secretion and assembly were investigated mostly by means of cellular hepatocyte model systems, yielded contrasting results and thus remain highly controversially discussed (see review [23]). Assembly of apo(a) and apoB to Lp(a) is generally viewed as a two-step procedure [24, 25]. In a first step, distinct domains within the apoB molecule initially associate with apo(a) in a non-covalent interaction to bring the two molecules into close proximity. In a second step, a disulfide bond is formed between apo(a) cysteine 4057 and apoB cysteine 4326 residues [24, 26]. Whether this disulfide bond is formed through a spontaneous oxidation reaction or through a specific enzymatic reaction is unclear $[27,28]$.

The location of this assembly process is the subject of controversial discussion as well. Intracellular, extracellular and/or plasma membrane-associated assembly procedures have been reported to occur in various cell systems [23]. Lp(a), like many other oligomeric protein complexes, may assemble in the endoplasmic reticulum of the hepatocyte and be secreted as a whole particle [29,30]. Alternatively, newly synthesized apo(a) could bind extracellularly to preexisting LDL or VLDL circulating in the plasma. Most authors postulate an extracellular assembly of $\mathrm{Lp}(\mathrm{a})$ based on studies conducted in various cellular model systems. White et al. could not detect an intracellular apo(a)-apoB complex by adding antiapo(a) antiserum to the culture medium of primary baboon hepatocytes, but found such complexes attached to the plasma membrane. The authors therefore concluded that, in that cellular system, $\mathrm{Lp}(\mathrm{a})$ is primarily assembled after secretion and to some extent also on the plasma membrane [31]. This conclusion has to be, however, critically evaluated since baboon hepatocytes secrete most of their apoB as VLDL, which does not associate with apo(a) [32]. Similar studies in apo(a)-transfected HepG2 cells could not demonstrate an intracellular apo(a)-apoB assembly for this human hepatocyte model and thus confirmed the results from the baboon studies [24, 33, 34]. Nevertheless, there is also evidence for intracellular assembly of $\mathrm{Lp}(\mathrm{a})$ in cell culture systems. Bonen et al. were able to detect an intracellular apo(a)-apoB complex in HepG2 cells transfected with an apo(a) minigene [35]. HepG2 cells have been reported to secrete a triglyceride-rich lipoprotein particle with an LDL density that does not exist at all in human plasma [36]. Taken together, the extracellular Lp(a) assembly proposed by numerous in vitro studies needs to be reviewed with caution, because these studies used cellular models that do not reflect the physiological lipoprotein metabolism.

\subsubsection{In vivo metabolism of $L p(a)$ and $L D L$ in healthy subjects}

Kinetic in vivo studies in humans have unfortunately also produced controversial results. Krempler et al. injected radiolabeled VLDL in Lp(a)-positive healthy probands and found no metabolic relationship between apoB in VLDL or LDL and apoB in Lp(a). The authors therefore concluded that $\mathrm{Lp}(\mathrm{a})$ seems to be synthesized as a separate lipoprotein 
independently of other apoB-containing lipoproteins [37, 38]. Two in vivo turnover studies using stable-isotope labeling techniques came to the same conclusion: Morrisett et al. and Su et al. observed similar synthesis rates of $\operatorname{Lp}(\mathrm{a})$-apo(a) and $\mathrm{Lp}(\mathrm{a})$-apoB [39, 40]. While these findings are compatible with an intracellular assembly of nascent apo(a) and apoB to $\operatorname{Lp}(\mathrm{a})$, two other kinetic studies concluded that Lp(a) originates from de novo hepatic LDL as well as from plasma LDL [41, 42].

We investigated by stable-isotope technology the metabolism of apo(a) and apoB-100, the two major Lp(a) protein components, in comparison to apoB of LDL in nine healthy probands. The metabolic data accumulating in this study after appropriate modeling present a scenario of virtually complete intracellular assembly of Lp(a) [43].

Mean FSR, RT and PR values of apo(a) from Lp(a) were similar to those of apoB from Lp(a) but significantly different from the kinetic parameters of LDL-apoB. The differences were particularly large between the PR values of LDL and $L p(a)$ since this parameter takes into account plasma concentrations that are much higher for LDL than for $\mathrm{Lp}(\mathrm{a})$.

Tracer/tracee data from Lp(a)-apo(a), Lp(a)-apoB, LDL-apoB and VLDL-apoB were analyzed based on the multicompartment model shown in Figure 3 in order to investigate whether Lp(a) assembles from circulating LDL or from de novo produced "hepatic" LDL. 92\% of leucine in $\mathrm{Lp}(\mathrm{a})$-apoB originated from the hepatic apoB pool. The remaining $8 \%$ derived from plasma LDL-apoB. LDL-apoB stemmed from two sources, namely from VLDL-apoB (54\%) and from de novo synthesis (46\%).

The kinetic parameters obtained from this in vivo turnover study of $\mathrm{Lp}$ (a) metabolism in healthy men allow three major conclusions: i) Since FSRs of both protein components of $\mathrm{Lp}(\mathrm{a})$ is very similar and different from those of LDL, an almost exclusive intracellular hepatic Lp(a) assembly can be assumed. This analysis, however, does not allow any conclusions to be drawn on where (inside the hepatocyte, at its plasma membrane or, eventually, in the space of Dissé) this assembly takes place. ii) Apo(a) FSR/FCR is positively related to the number of apo(a) kringle 4 repeats (e.g. apo(a) molecular size), suggesting that plasma $\mathrm{Lp}$ (a) concentrations are controlled not only by synthesis but also to some smaller extent by degradation. iii) Longer plasma RT of apo(a) from probands with LMW apo(a) isoforms compared to those with HMW apo(a) isoforms help to explain the potential atherogenicity of higher concentrations in carriers with LMW apo(a) isoforms.

The de novo synthesis of LDL is an absolute prerequisite for the postulated (intra)cellular hepatic assembly of Lp(a). Such a "direct" LDL production has been questioned by some investigators who presume that it may instead be the consequence of a very fast lipolytic pathway [45]. However, metabolic studies of apoB metabolism using stable-isotope technology fitted by multicompartmental modeling support a significant "direct" LDL production by the liver [46, 47]. A substantial amount of nascent LDL production was also detected in cultured primary human hepatocytes [48] but not in HepG2 cells [36]. Lp(a) secretion was previously demonstrated in such cells, thus additionally supporting the view of "direct" LDL synthesis by human hepatocytes [49]. 


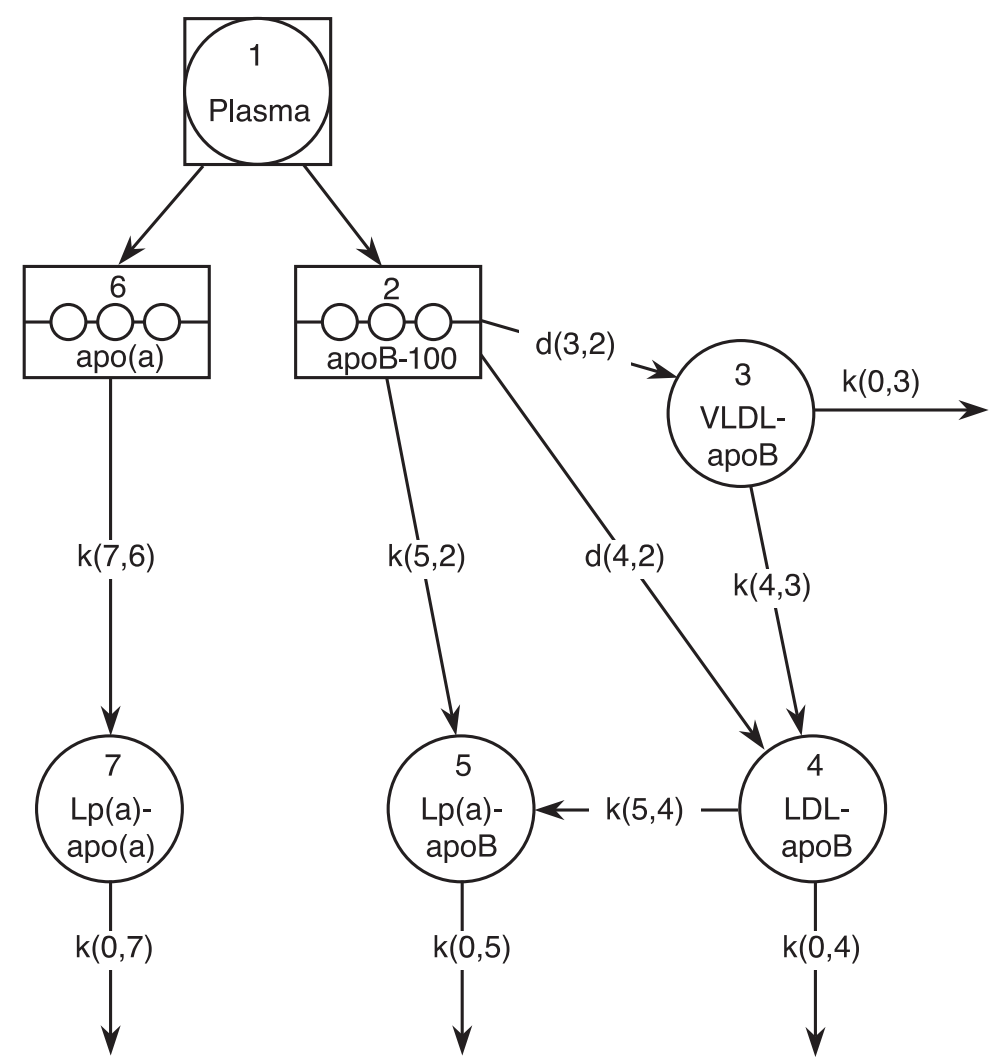

Figure 3. Multicompartmental model for apoB-100 and apo(a) metabolism. A plasma leucine pool (compartment 1) was used as a forcing function, and delay compartments that account for assembly and subsequent secretion of apoB-100 (compartment 2) and apo(a) (compartment 6), respectively. ApoB in VLDL, LDL, Lp(a), and apo(a) in Lp(a) consist all of single compartments. The input of apoB in Lp(a) is twofold: one via de novo synthesis from the liver and one from LDL-apoB. $\mathrm{d}(\mathrm{i}, \mathrm{j})$ denotes the distribution of transfer from the delay compartment $j$ to compartment $i$ and $k(i, j)$ represents the rate constant from compartment $j$ to compartment $i$. In this model, tracer/tracee data for VLDL, LDL, Lp(a) apoB, and Lp(a) apo(a) as well as leucine masses $(\mathrm{nmol} / \mathrm{L})$ in these compartments were fitted simultaneously. The fractional catabolic rate (FCR) of apo(a)-Lp(a) and apoB-Lp(a) was equal to $\mathrm{k}(0,7)$ and $\mathrm{k}(0,5)$, respectively (taken from Frischmann et al. [44], with permission).

\subsection{Lipoprotein metabolism in HD patients suffering from chronic kidney disease (CKD)}

Dyslipidemia in patients with CKD and hemodialysis (HD) patients is distinct from other organ-specific diseases with far-reaching therapeutic consequences (see review [2]). It involves all lipoprotein classes, shows considerable variations depending on the stage of CKD [50, 51] and is further modified by concurrent diseases such as diabetes [52] and nephrotic syndrome [53]. In addition, major qualitative compositional changes in lipoprotein particles, such as oxidation, glycation, carbamylation and formation of small dense LDL (sdLDL - see below) 
which render the particles more atherogenic, have been observed [54]. Reduced activities of plasma cholesterol esterification and cholesterol ester transfer between lipoproteins - key factors for the so-called "reversed cholesterol transport" - result in substantially abnormal lipid composition of virtually all lipoprotein classes in HD patients [55].

Plasma triglycerides start to increase in early stages of CKD and show the highest concentrations in nephrotic syndrome and in patients treated with peritoneal dialysis (PD). In pre-dialysis CKD patients, the accumulation of triglycerides is the consequence of both an increased PR and a decreased FCR of triglyceride-rich lipoproteins [56]. The increased production of triglyceride-rich lipoproteins is possibly a consequence of impaired carbohydrate tolerance and enhanced hepatic VLDL synthesis [57]. The reduced catabolism is likely due to decreased activities of LPL and HL [58,59], two endothelium-associated lipases that cleave triglycerides into free fatty acids for energy production or storage.

Diminished catabolism results in the accumulation of IDL particles contributing to compositional and size heterogeneity of triglyceride-rich lipoproteins in plasma of CKD patients. IDL are rich in apoE, a ligand that is important for removal from the circulation by binding to the LDL receptor [60]. The arterial wall therefore is exposed to high plasma concentrations of IDL which may predispose to atherosclerosis [54].

Elevated plasma concentrations of LDL cholesterol and -apoB are common in nephrotic syndrome and PD but do not occur in patients with advanced CKD, treated with HD. There are, however, qualitative changes in LDL in patients with CKD and dialysis patients. The fraction of sdLDL, which is considered to be highly atherogenic, is increased in HD patients. sdLDL is a subtype of LDL which penetrates the vessel wall more efficiently than normal LDL, becomes oxidized, and triggers atherosclerotic processes. In addition, sdLDL exert a high affinity for macrophages promoting their entry into the vascular wall to participate in the formation of foam cells and atherosclerotic plaques [61].

In kidney disease, elevated plasma Lp(a) concentrations are not only genetically determined but also a consequence of kidney failure [62]. In predialysis CKD patients, Lp(a) concentrations are influenced by the glomerular filtration rate (GFR). In patients with HMW apo(a) isoforms but not in those with LMW apo(a) isoforms, plasma Lp(a) concentrations begin to increase in stage 1 CKD before GFR starts to decrease [50]. This isoform-specific increase in plasma Lp(a) concentrations was observed in several but not all studies in CKD and HD patients [50, 62-66]. In contrast, in patients with nephrotic syndrome [67, 68] and in PD patients [63], increases in plasma Lp(a) concentrations occur in all apo(a) isoform groups, probably as a consequence of the pronounced protein loss and a subsequently increased production in the liver [69]. After successful kidney transplantation, a decrease in plasma $\mathrm{Lp}(\mathrm{a})$ can be observed in HD patients with HMW apo(a) isoforms [70, 71] and in PD patients with all apo(a) isoform groups [72]. Thus, the elevation of $\mathrm{Lp}(\mathrm{a})$ in CKD is due to non-genetic causes, mostly influenced by the degree of proteinuria [50,67] and less by the cause of kidney disease [63].

In summary, the hallmarks of uremic dyslipidemia include hypertriglyceridemia and increased circulating concentrations of IDL, sdLDL and Lp(a). HD patients are characterised by normal LDL concentrations, whereas patients with nephrotic syndrome and CKD patients treated by PD are diagnosed with elevated LDL concentrations. 


\subsubsection{Dyslipidemia and CVD in CKD}

Forty years ago, Lindner and colleagues described in their seminal report the excessive risk of CVD in HD patients for the first time [73]. Later, Foley et al. extended these observations by reporting a 10 to 20 times higher mortality rate in HD patients compared to the general population [8]. While in the general population high plasma concentrations of apoBcontaining lipoproteins, low concentrations of HDL cholesterol and high total triglyceride concentrations are associated with an increased atherosclerotic cardiovascular risk [74], most investigations, including cross-sectional [75-78] and longitudinal [66, 79-87] studies, do not support the association between dyslipidemia and CVD in hemodialysed CKD populations or even observe opposite associations. Indeed, a worse survival among HD patients has been observed with low rather than high BMI [88], blood pressure [89] and serum/plasma concentrations of cholesterol [90]. This seemingly paradoxical phenomenon is often called „reverse epidemiology“ [91] and exemplified in crossing curves when relating BMI with mortality in HD patients and the general population [92].

While the BMI-associated death risk shows an almost linear negative gradient in HD patients [92], the relationship between plasma total cholesterol and mortality has been found to be U-shaped [93]. The group with total cholesterol between 200 and $250 \mathrm{mg} / \mathrm{dl}$ had the lowest risk for death, whereas those with levels $>350 \mathrm{mg} / \mathrm{dl}$ had a relative risk of 1.3 -fold and those with levels $<100 \mathrm{mg} / \mathrm{dl}$ had a relative risk of 4.2 -fold. The association between low total cholesterol and increased mortality, however, was reduced after statistical adjustment for plasma albumin levels. This dichotomous relationship was confirmed in the Choices for Healthy Outcomes in Caring for ESRD (CHOICE) study [94], which showed a nonsignificant negative association of cardiovascular mortality with plasma total as well as non-HDL cholesterol levels in the presence of inflammation and/or malnutrition; in contrast, there was a positive association between total and non-HDL cholesterol and mortality in the absence of inflammation or malnutrition. These observations are compatible with the hypothesis that the inverse association of total cholesterol levels with mortality in dialysis patients is mediated by the cholesterol-lowering effect of malnutrition and/or systemic inflammation and not due to a protective effect of high cholesterol concentrations.

The association of $\mathrm{Lp}(\mathrm{a})$ with atherosclerotic complications and CVD has been investigated in numerous studies in dialysis patients. Like other atherogenic lipoproteins, Lp(a) has been found to contribute to the high cardiovascular burden [66, 79, 84, 95-97]. When apo(a) phenotyping was performed along with plasma $\mathrm{Lp}(\mathrm{a})$ concentrations, an association between the apo(a) K-IV repeat polymorphism and CV complications was consistently observed.

Two final considerations regarding the impact of classical risk factors for the development of CVD in CKD patients are, however, worth mentioning: the cardiovascular risk for an individual CKD patient at a given time point is the sum (or combination) of risk exposure before and after developing CKD. When taking Lp(a) concentrations and apo(a) isoforms as an example, a previously healthy subject with low Lp(a) concentrations and a HMW apo(a) isoform develops CKD with subsequently rising $\mathrm{Lp}(\mathrm{a})$ concentrations covering a relatively 
short period of his lifespan. A subject with LMW apo(a) isoform, on the other hand, has genetically caused elevated $\mathrm{Lp}$ (a) concentrations for his whole life which do not substantially increase after developing CKD. Since the HMW apo(a) carrier is exposed to elevated atherogenic $\mathrm{Lp}(\mathrm{a})$ for a much shorter period of his life, this condition has to be considered less CVD-prone than having LMW apo(a). This example demonstrates the importance of the "longitudinal“ factor when considering risk factors for CVD in CKD patients.

Finally, as already discussed in the introduction, the quantification of a target parameter deemed to be associated with or predictive for a disease can only provide a static picture and hardly reflects the true in vivo metabolism. Seemingly normal blood concentrations of suspected marker candidates can only be validated by kinetic studies in humans and have been therefore performed also in CKD patients. They have provided novel and unexpected information regarding the physiology and pathology of atherogenic apoB-containing lipoproteins (see review [98]).

\subsubsection{Delayed in vivo catabolism of LDL and IDL in HD patients as potential cause of premature atherosclerosis}

For better understanding the atherogeneity of apoB-containing lipoproteins in HD patients and to resolve the apparent discrepancy between their obviously impaired lipoprotein metabolism and e.g. normal LDL plasma concentrations, we studied the in vivo kinetics of VLDL, IDL and LDL by stable isotope technology in HD patients and compared them to those of healthy controls [12].

This study demonstrated for the first time severely decreased FCRs of IDL- and LDL-apoB in HD patients as compared to controls (Figure 4), whereas the in vivo kinetics of VLDL did not change significantly. A decreased FCR of IDL- and LDL-apoB is identical to a prolonged RT of these highly atherogenic particles. The longer RT of these lipoproteins results in an extended exposure to oxidation for IDL and LDL in a highly oxidative environment. This is in line with experimental data showing a highly significant correlation of 5-hydroxy-2aminovaleric acid (HAVA) in LDL, an oxidation product of apoB, with LDL RT in normolipidemic controls [99]. In accordance with these results, two previously conducted randomized placebo-controlled studies revealed a significant reduction in composite cardiovascular disease endpoints when HD patients were treated for two years with supplementation of antioxidants such as vitamin E [100] or acetylcysteine [101].

Most remarkably, the observed impaired metabolism of apoB-containing lipoproteins is accompanied by normal concentrations of LDL-apoB and elevated levels of IDL-apoB (Figure 4), in line with previous reports which found increased concentrations of IDL as an independent risk factor for atherosclerosis in HD patients [102]. A closer look at the kinetic data reveals that the normal concentrations of LDL are the result of a combination of decreased FCR and PR. This pattern therefore demonstrates convincingly the strength of kinetic studies in contrast to simply quantifying blood concentrations of a target marker such as LDL concentrations. Its normal concentrations are masked by two metabolic disorders which neutralise each other and result in normal values such as observed in the 
general population. The altered lipoprotein metabolism therefore puts HD patients at high risk for developing atherosclerotic disease despite their normal total and LDL cholesterol concentrations. Since most lipid-lowering drugs act by "normalising" the RT of the major atherogenic lipoproteins IDL and LDL [103], these drugs are expected to correct some of the basic defects of the severely disturbed lipoprotein metabolism in HD patients. Therefore, kinetic studies on the impact of lipid-lowering medication on the lipoprotein metabolism in CKD patients were a logic consequence of the observed, above-described findings (see chapter 3.2.5.).

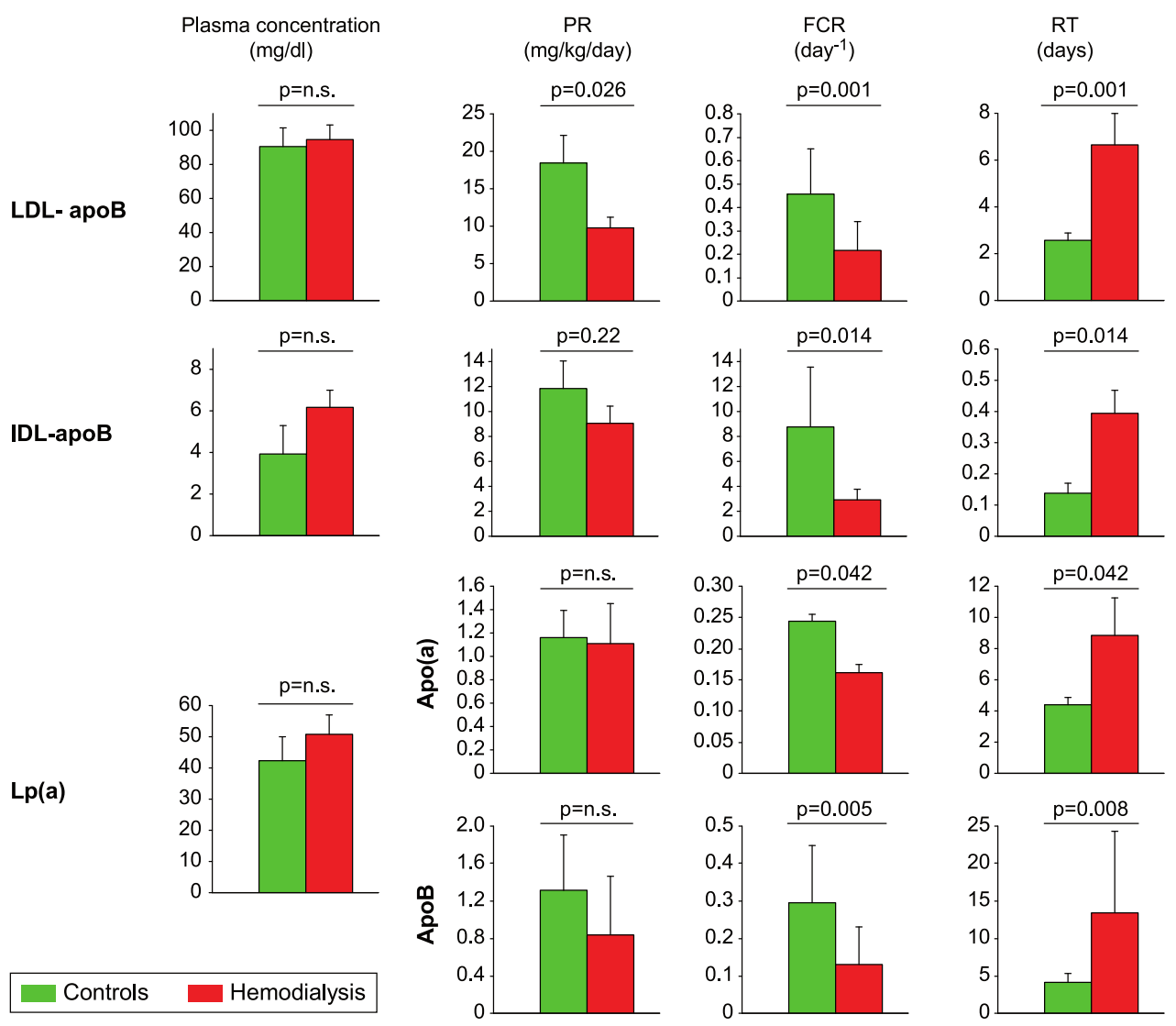

Figure 4. Kinetic parameters of apoB in LDL, IDL and Lp(a) and apo(a) in Lp(a). Plasma concentrations, production rates (PR), fractional catabolic rates (FCR) and residence times (RT) are given for healthy controls (green columns) and HD patients (red columns). Columns represent mean values \pm SD. Results for LDL and IDL are taken from Ikewaki et al. [12], those for Lp(a) from Frischmann et al. [44].

Due to the laborious nature of these studies and the complexity of the metabolic modeling, only few studies have been performed so far in CKD patients either by radiotracer or stable isotope technology. Our kinetic data seem to contrast with a previously published turnover study in Finnish HD patients performed with conventional radiotracer techniques. While 
the authors found decreased LDL clearance rates in predialysis CKD patients [104] they could not find a significant difference in LDL-apoB FCR between HD patients and controls [105]. More recently, Prinsen et al., by using stable isotopes, found unchanged FCRs for LDL-apoB in CKD patients treated with peritoneal dialysis [106]. Chan et al. injected radiolabeled VLDL into HD patients with or without hyperlipidemia and found decreased FCRs of VLDL-apoB and IDL-apoB (the latter only in hyperlipidemic patients) [107]. LDL kinetics were not investigated in this study. The reason for these discrepancies is not clear. There might be ethnic differences in the lipoprotein metabolism between the investigated patient populations of different ethnic origin. One major difference between our and the Finnish study is an age difference between patients and controls in our but not in the Finnish study. Our control subjects were considerably younger than the HD patients ( 35 vs. 51 years). At first glance, this age difference might explain to some extent the dramatic differences found in our study, since LDL clearance rates have been repeatedly described to decrease with age presumably due to down-regulated hepatic LDL receptor expression in the elderly [108, 109]. Based on the results of these studies, an age difference of 15 years (as observed in our work) would result in an approximately $10 \%$ change in FCR values and could therefore not explain the more than two-fold difference in our study. The observed differences in kinetic parameters can therefore not be explained by age differences between study groups.

Several mechanisms may contribute to our observations. First, the diminished LDL catabolism in HD patients might be explained by a possible contribution of LDL uptake by the healthy human kidney which does not function appropriately (or at all) in chronic kidney failure. In fact, glomerular cells like mesangial or epithelial cells have been shown in vitro to express lipoprotein receptors and to take up LDL comparably to fibroblasts and hepatocytes [110]. It is, however, completely unclear whether the kidney plays a significant role in LDL catabolism in vivo. Perfusion studies in rat kidneys indicate that virtually no intact LDL is cleared from the circulation by the kidney [111]. Second, an impaired lipolytic cascade in HD patients most likely also contributes to our results. The relatively normal VLDL concentrations and kinetic parameters and the correspondingly impaired IDL parameters are in good accordance with previous findings of normal lipoprotein lipase (LPL) but significantly decreased activities of hepatic triglyceride lipase (HL) in HD patients [59]. Since HL promotes the conversion of IDL to LDL, a decrease in HL activity might contribute to the accumulation of IDL and reduced production rates of LDL (without accumulating small, dense LDL) in HD patients.

\subsubsection{Kinetics of $\operatorname{Lp}(a)$ in hemodialysis patients}

We previously performed in vivo kinetic studies using stable-isotope techniques to elucidate the mechanism for increased plasma Lp(a) concentrations in HD patients [44]. PRs of apo(a) and apoB, the two apolipoproteins contained in $\mathrm{Lp}(\mathrm{a})$, were normal, when compared to control subjects with similar plasma Lp(a) concentrations (Figure 4). The FCR of these apolipoproteins was, however, significantly reduced compared to controls resulting in a much longer plasma RT for apo(a) of almost 9 days, compared to only 4.4 days in controls. Since the PR of Lp(a) did not differ between HD patients and controls, its decreased 
clearance in HD patients leads to increased Lp(a) plasma concentrations and is likely the result of loss in kidney function [44]. A role of the kidney in the catabolism has been previously supported by the observation of renovascular arteriovenous differences in $\mathrm{Lp}(\mathrm{a})$ concentrations [112] as well as apo(a) fragments in urine [113, 114].

Comparing kinetic data in HD patients [44] with those in patients with nephrotic syndrome [69] points to fundamental differences in the metabolism of $L p(a)$ and other proteins between these two patient groups. Patients with nephrotic syndrome do not differ with respect to the FCR of $\mathrm{Lp}(\mathrm{a})$ compared to controls but have increased Lp(a) PRs [69]. It is well known that nephrotic patients show a generally increased lipoprotein synthesis of lipoproteins [115]. Since kidney function is relatively well preserved in nephrotic syndrome, a decreased clearance of $\mathrm{Lp}(\mathrm{a})$ in these patients is not likely to be expected. Metabolic differences between nephrotic and dialysis patients are not only evident for $L p(a)$ but also for albumin. Whereas the FCR of albumin in HD patients is similar or even reduced compared to controls, the FCR in patients with nephrotic syndrome is increased [116, 117].

\subsubsection{Consequences of the impaired metabolism of atherogenic lipoproteins in HD patients}

The observation of markedly decreased FCRs of apoB of LDL and IDL as well as apo(a) and apoB in $\mathrm{Lp}(\mathrm{a})$ causes a prolonged RT of these highly atherogenic lipoproteins. Due to the long retention period, "aged" lipoprotein complexes are thus more susceptible for alterations such as oxidation damage, which was shown to be associated with accelerated atherogenesis in HD patients [118]. Previous kinetic studies investigated the metabolism of the two LDL subclasses, "buoyant" LDL1 and the smaller cholesterol-poor "dense" LDL2, in subjects with familial defective apoB-100 (FDB). The authors found a more than four-fold longer RT for small dense LDL2 in those patients as compared to normolipidemic controls [99]. It was therefore suggested that oxidative damage of an "aged" LDL2, which is present in large concentrations in both blood and the subendothelial space, may be an important mechanism for the development of premature atherosclerosis in patients with familial defective apoB-100. Since the LDL-like particle of $L p(a)$ is compositionally similar to LDL2 [41], it is tempting to speculate that the increased RT of circulating Lp(a) might pose an additional risk factor for the increased incidence of cardiovascular disease in HD patients.

\subsubsection{Influence of statin treatment on kinetic parameters in hemodialysed patients}

In the general population, therapy with HMG-CoA-reductase inhibitors (statins) which inhibit endogenous cholesterol biosynthesis has shown to improve outcome in several atherosclerotic diseases [119, 120]. The inhibition of cholesterol biosynthesis subsequently leads to up-regulation of LDL receptors and therefore increased clearance and thus reduced RT of circulating LDL [103]. Statins also have a beneficial role as antiinflammatory agents, which is independent of their lipid-lowering effect. Inflammation is highly prevalent in patients with CKD and is consistently associated with cardiovascular morbidity and mortality. In line with this metabolic background, the first studies in HD patients demonstrated a substantial normalisation of the dyslipidemic plasma profile and 
reduced progression of renal disease [121, 122] and in one study also reduced mortality [123] in these patients.

In contrast and quite surprisingly, three previously conducted large, randomized, placebocontrolled trials on statin treatment in CKD patients had not led to significant benefits regarding their primary cardiovascular outcome. Two of those studies, the German Diabetes Dialysis (4D) Study and the Study to Evaluate the Use of Rusovastatin in Subjects on Regular Hemodialysis (AURORA) were performed on HD patients, one study, the Assessment of Lescol in Renal Transplantation (ALERT) Study on patients who had undergone kidney transplantation. Their primary endpoints (death of cardiovascular cause, nonfatal myocardial infarction or nonfatal stroke) were virtually unchanged [124-126]. However, there has been a promising risk reduction in the secondary endpoint 'all cardiac events combined' in one study [126]. A simulated study of exactly the same trial using a large historical database with more than 10.000 patients also demonstrated that statin use was associated with some benefit [127]. A comprehensive review of outcome data from the $4 \mathrm{D}$ and AURORA trials found no benefit of statin therapy in either the whole study group of HD patients or after stratification for inflammatory marker levels [128]. More recently, another, much larger trial including 9270 patients with chronic kidney disease, the Study of Heart and Renal Protection (SHARP) could show a significant risk reduction in cardiovascular events in a mixed population of patients with kidney disease including $2 / 3$ predialysis and 1/3 HD patients treated with a combination of a statin and ezitimibe. This effect did not differ between HD and predialysis patients [129].

Based on our previous studies on lipoprotein kinetics in HD patients and the abovedescribed conflicting results regarding their cardiovascular risk profile after statin treatment, we examined by stable-isotope technology the in vivo kinetics of apoB-containing particles in HD patients before and after treatment with atorvastatin (Schwaiger et al., unpublished).

In this study we described for the first time effects of HMG-CoA reductase inhibition on apoB metabolism in CKD patients treated with HD. Low-dose atorvastatin, given for three months to six male patients, lowered, as expected, concentrations of VLDL- and LDL-apoB, both accompanied by a significant increase of their FCR, while hepatic production of both apolipoproteins was not altered. This led, as expected, to a lower RT of these atherogenic apoB-containing particles comparable to RT values of healthy subjects with normal kidney function. The observed findings therefore argue for a beneficial effect of statin therapy regarding cardiovascular events in HD patients similar to described for the general population.

To understand why statins have surprisingly failed to reduce cardiovascular events in HD patients, the basic mechanisms underlying the pathophysiology of CVD in CKD must be critically considered. In contrast to the general population, CKD patients suffer, in addition to dyslipidemia, from several further complex comorbid conditions including diabetes mellitus, hypertension, oxidative stress, inflammation, insulin resistance, anemia and disturbances in mineral metabolism. Lipid lowering therapy by statins have the potential to 
ameliorate only some but no all of those conditions (see review [130]). Taken together, statin therapy in CKD maybe recommended based on our kinetic studies on apoB-containing lipoproteins, optimally combined with medication to treat atherogenic non-lipid factors in HD patients.

\section{Conclusion}

Kinetic in vivo studies in human subjects are superior to many methodological approaches including animal and cell culture models and thus represent the ultimate approach to understand basic metabolic pathways in humans. They have clearly revolutionized human lipoprotein research and have particularly resulted in novel insights into the metabolism of atherogenic apoB-containing lipoproteins some of which have been the subject of our previous investigations and object of this review.

\section{Author details}

Benjamin Dieplinger*

Department of Laboratory Medicine, Konventhospital Barmherzige Brüder Linz, Austria

Hans Dieplinger ${ }^{* *}$

Division of Genetic Epidemiology, Department of Human Genetics and Molecular Pharmacology, Medical University of Innsbruck, Austria

\section{Acknowledgement}

We would like to thank Ramona Berberich, Linda Fineder, Michael E. Frischmann, Tatsuo Hosoya, Katsunori Ikewaki, Paul König, Florian Kronenberg, Seibu Mochizuki, Yoshinobu Nakada, Ulrich Neyer, Keio Okubo, Hermann Salmhofer Jürgen R. Schäfer, Johannes P. Schwaiger, Horst Schweer, Alex Starke, Evi Trenkwalder and Emanuel Zitt for invaluable contributions over many years within the various projects covered in this chapter. Funding support from the Austrian Science Fund (P10090-MED, P12358-MED), the Austrian National Bank $(6721 / 4,9331)$ and Pfizer-Austria (ATV-A-02-007G) is greatly appreciated.

\section{References}

[1] Mahley RW, Innerarity TL, Rall SCJ, Weisgraber KH. Plasma lipoproteins: Apolipoprotein structure and function. J Lipid Res 1984;25:1277-1294.

[2] Kwan BC, Kronenberg F, Beddhu S, Cheung AK. Lipoprotein metabolism and lipid management in chronic kidney disease. J Am Soc Nephrol 2007;18:1246-1261.

[3] Williams KJ, Tabas I. The response-to-retention hypothesis of early atherogenesis. Arterioscler Thromb Vasc Biol 1995;15:551-561.

\footnotetext{
* benjamin.dieplinger@bs-lab.at

** Corresponding Author, hans.dieplinger@i-med.ac.at
} 
[4] Boren J, Taskinen MR, Adiels M. Kinetic studies to investigate lipoprotein metabolism. J Intern Med 2012;271:166-173.

[5] Adiels M, Olofsson SO, Taskinen MR, Boren J. Overproduction of very low-density lipoproteins is the hallmark of the dyslipidemia in the metabolic syndrome. Arterioscler Thromb Vasc Biol 2008;28:1225-1236.

[6] Jawien J, Nastalek P, Korbut R. Mouse models of experimental atherosclerosis. J Physiol Pharmacol 2004;55:503-517.

[7] Javitt NB. Hep G2 cells as a resource for metabolic studies: Lipoprotein, cholesterol, and bile acids. FASEB J 1990;4:161-168.

[8] Foley RN, Parfrey PS, Sarnak MJ. Clinical epidemiology of cardiovascular disease in chronic renal disease. Am J Kidney Dis 1998;32:S112-119.

[9] Krempler F, Kostner GM, Bolzano K, Sandhofer F. Turnover of lipoprotein(a) in man. J Clin Invest 1980;65:1483-1490.

[10] Barrett PH, Chan DC, Watts GF. Thematic review series: patient-oriented research. Design and analysis of lipoprotein tracer kinetics studies in humans. J Lipid Res 2006;47:1607-1619.

[11] Ikewaki K, Rader DJ, Sakamoto T, et al. Delayed catabolism of high density lipoprotein apolipoproteins A-I and A-II in human cholesteryl ester transfer protein deficiency. J Clin Invest 1993;92:1650-1658.

[12] Ikewaki K, Schaefer JR, Frischmann ME, et al. Delayed in vivo catabolism of intermediate-density lipoprotein and low-density lipoprotein in hemodialysis patients as potential cause of premature atherosclerosis. Arterioscler Thromb Vasc Biol 2005;25:2615-2622.

[13] Barrett PH, Bell BM, Cobelli C, et al. SAAM II: Simulation, analysis, and modeling software for tracer and pharmacokinetic studies. Metabolism 1998;47:484-492.

[14] Cobelli C, Foster DM. Compartmental models: theory and practice using the SAAM II software system. Adv Exp Med Biol 1998;445:79-101.

[15] Utermann G. Lipoprotein(a). In: Scriver C. R., Beaudet A. L., Sly W. S.,Valle D. (eds), The metabolic bases of inherited disease, New York, McGraw Hill Inc., 2001:2753-2787.

[16] Clarke R, Peden JF, Hopewell JC, et al. Genetic variants associated with Lp(a) lipoprotein level and coronary disease. N Engl J Med 2009;361:2518-2528.

[17] Kraft HG, Köchl S, Menzel HJ, Sandholzer C, Utermann G. The apolipoprotein(a) gene: a transcribed hypervariable locus controlling plasma lipoprotein(a) concentration. Hum Genet 1992;90:220-230.

[18] Schmidt K, Kraft HG, Parson W, Utermann G. Genetics of the Lp(a)/apo(a) system in an autochthonous Black African population from the Gabon. Eur J Hum Genet 2006;14:190201.

[19] Bostom AG, Gagnon DR, Cupples LA, et al. A prospective investigation of elevated lipoprotein (a) detected by electrophoresis and cardiovascular disease in women: The Framingham Heart Study. Circulation 1994;90:1688-1695.

[20] Erqou S, Kaptoge S, Perry PL, et al. Lipoprotein(a) concentration and the risk of coronary heart disease, stroke, and nonvascular mortality. JAMA 2009;302:412-423. 
[21] Rader DJ, Cain W, Ikewaki K, et al. The inverse association of plasma lipoprotein(a) concentrations with apolipoprotein(a) isoform size is not due to differences in Lp(a) catabolism but to differences in production rate. J Clin Invest 1994;93:2758-2763.

[22] Dube JB, Boffa MB, Hegele RA, Koschinsky ML. Lipoprotein(a): more interesting than ever after 50 years. Curr Opin Lipidol 2012;23:133-140.

[23] Dieplinger H, Utermann G. The seventh myth of lipoprotein(a): where and how is it assembled? Curr Opin Lipidol 1999;10:275-283.

[24] Brunner C, Kraft HG, Utermann G, Müller HJ. Cys4057 of apolipoprotein(a) is essential for lipoprotein(a) assembly. Proc Natl Acad Sci U.S.A. 1993;90:11643-11647.

[25] Trieu VN, McConathy WJ. A two-step model for lipoprotein(a) formation. J Biol Chem 1995;270:15471-15474.

[26] McCormick SP, Ng JK, Taylor S, Flynn LM, Hammer RE, Young SG. Mutagenesis of the human apolipoprotein B gene in a yeast artificial chromosome reveals the site of attachment for apolipoprotein(a). Proc Natl Acad Sci U.S.A. 1995;92:10147-10151.

[27] Becker L, Cook PM, Koschinsky ML. Identification of sequences in apolipoprotein(a) that maintain its closed conformation: a novel role for apo(a) isoform size in determining the efficiency of covalent Lp(a) formation. Biochemistry 2004;43:9978-9988.

[28] Becker L, Nesheim ME, Koschinsky ML. Catalysis of covalent Lp(a) assembly: evidence for an extracellular enzyme activity that enhances disulfide bond formation. Biochemistry 2006;45:9919-9928.

[29] Gething MJ, Sambrook J. Protein folding in the cell. Nature 1992;355:33-45.

[30] Hurtley SM, Helenius A. Protein oligomerization in the endoplasmic reticulum. Annu Rev Cell Biol 1989;5:277-307.

[31] White AL, Lanford RE. Biosynthesis and metabolism of lipoprotein(a). Curr Opin Lipidol 1995;6:75-80.

[32] White AL, Rainwater DL, Lanford RE. Intracellular maturation of apolipoprotein[a] and assembly of lipoprotein[a] in primary baboon hepatocytes. J Lipid Res 1993;34:509-517.

[33] Lobentanz EM, Krasznai K, Gruber A, et al. Intracellular metabolism of human apolipoprotein(a) in stably transfected Hep G2 cells. Biochemistry 1998;37:5417-5425.

[34] Koschinsky ML, Côté GP, Gabel B, Van der Hoek YY. Identification of the cysteine residue in apolipoprotein(a) that mediates extracellular coupling with apolipoprotein B100. J Biol Chem 1993;268:19819-19825.

[35] Bonen DK, Hausman AML, Hadjiagapiou C, Skarosi SF, Davidson NO. Expression of a recombinant apolipoprotein(a) in HepG2 cells. Evidence for intracellular assembly of lipoprotein(a). J Biol Chem 1997;272:5659-5667.

[36] Dashti N, Alaupovic P, Knight-Gibson C, Koren E. Identification and partial characterization of discrete Apolipoprotein B containing lipoprotein particles produced by human hepatoma cell line HepG2. Biochemistry 1987;26:4837-4846.

[37] Krempler F, Kostner GM, Bolzano K, Sandhofer F. Lipoprotein(a) is not a metabolic product of other lipoproteins containing apolipoprotein B. Biochim Biophys Acta 1979;575:63-70.

[38] Krempler F, Kostner G, Bolzano K, Sandhofer F. Studies on the metabolism of the lipoprotein Lp(a) in man. Atherosclerosis 1978;30:57-65. 
[39] Morrisett J, Gaubatz J, Nava L, et al. Metabolism of apo(a) and apoB-100 in human lipoprotein(a). In: Catapano A., Gotto Jr. A. M., Smith L. C.,Paoletti R. (eds), Drugs affecting lipid metabolism, Dordrecht, The Netherlands, Kluwer Academic Publishers and Fondazione Giovanni Lorenzini, 1993:161-167.

[40] Su W, Campos H, Judge H, Walsh BW, Sacks FM. Metabolism of Apo(a) and ApoB100 of lipoprotein(a) in women: effect of postmenopausal estrogen replacement. J Clin Endocrinol Metab 1998;83:3267-3276.

[41] Demant T, Seeberg K, Bedynek A, Seidel D. The metabolism of lipoprotein(a) and other apolipoprotein B-containing lipoproteins: a kinetic study in humans. Atherosclerosis 2001;157:325-339.

[42] Jenner JL, Seman LJ, Millar JS, et al. The metabolism of apolipoproteins (a) and B-100 within plasma lipoprotein (a) in human beings. Metabolism 2005;54:361-369.

[43] Frischmann ME, Ikewaki K, Trenkwalder E, et al. In vivo stable-isotope kinetic study suggests intracellular assembly of lipoprotein(a). Atherosclerosis 2012;(in press).

[44] Frischmann ME, Kronenberg F, Trenkwalder E, et al. In vivo turnover study demonstrates diminished clearance of lipoprotein(a) in hemodialysis patients. Kidney Int 2007;71:1036-1043.

[45] Shames DM, Havel RJ. De novo production of low density lipoproteins: Fact or fancy. J Lipid Res 1991;32:1099-1112.

[46] Pietzsch J, Wiedemann B, Julius U, et al. Increased clearance of low density lipoprotein precursors in patients with heterozygous familial defective apolipoprotein B-100: a stable isotope approach. J Lipid Res 1996;37:2074-2087.

[47] Packard CJ, Demant T, Stewart JP, et al. Apolipoprotein B metabolism and the distribution of VLDL and LDL subfractions. J Lipid Res 2000;41:305-318.

[48] Bouma ME, Pessah M, Renaud G, Amit N, Catala D, Infante R. Synthesis and secretion of lipoproteins by human hepatocytes in culture. In Vitro Cell Dev Biol 1988;24:85-90.

[49] Kagawa A, Azuma H, Akaike M, Kanagawa Y, Matsumoto T. Aspirin reduces apolipoprotein(a) (apo(a)) production in human hepatocytes by suppression of apo(a) gene transcription. J Biol Chem 1999;274:34111-34115.

[50] Kronenberg F, Kuen E, Ritz E, et al. Lipoprotein(a) serum concentrations and apolipoprotein(a) phenotypes in mild and moderate renal failure. J Am Soc Nephrol 2000;11:105-115.

[51] Kronenberg F, Kuen E, Ritz E, et al. Apolipoprotein A-IV serum concentrations are elevated in patients with mild and moderate renal failure. J Am Soc Nephrol 2002;13:461-469.

[52] Krentz AJ. Lipoprotein abnormalities and their consequences for patients with type 2 diabetes. Diabetes Obes Metab 2003;5 Suppl 1:S19-27.

[53] Kronenberg F. Dyslipidemia and nephrotic syndrome: recent advances. J Ren Nutr 2005;15:195-203.

[54] Shoji T, Ishimura E, Inaba M, Tabata T, Nishizawa Y. Atherogenic lipoproteins in endstage renal disease. Am J Kidney Dis 2001;38:S30-33. 
[55] Dieplinger H, Schoenfeld PY, Fielding CJ. Plasma cholesterol metabolism in end-stage renal disease. Difference between treatment by hemodialysis or peritoneal dialysis. J Clin Invest 1986;77:1071-1083.

[56] Batista MC, Welty FK, Diffenderfer MR, et al. Apolipoprotein A-I, B-100, and B-48 metabolism in subjects with chronic kidney disease, obesity, and the metabolic syndrome. Metabolism 2004;53:1255-1261.

[57] Appel G. Lipid abnormalities in renal disease. Kidney Int 1991;39:169-183.

[58] Chan MK, Persaud J, Varghese Z, Moorhead JF. Pathogenic roles of post-heparin lipases in lipid abnormalities in hemodialysis patients. Kidney Int 1984;25:812-818.

[59] Oi K, Hirano T, Sakai S, Kawaguchi Y, Hosoya T. Role of hepatic lipase in intermediatedensity lipoprotein and small, dense low-density lipoprotein formation in hemodialysis patients. Kidney Int Suppl 1999;71:S227-228.

[60] Brown MS, Goldstein JL. A receptor-mediated pathway for cholesterol homeostasis. Science 1986;232:34-47.

[61] Littlewood TD, Bennett MR. Apoptotic cell death in atherosclerosis. Curr Opin Lipidol 2003;14:469-475.

[62] Dieplinger H, Lackner C, Kronenberg F, et al. Elevated plasma concentrations of lipoprotein(a) in patients with end-stage renal disease are not related to the size polymorphism of apolipoprotein(a). J Clin Invest 1993;91:397-401.

[63] Kronenberg F, König P, Neyer U, et al. Multicenter study of lipoprotein(a) and apolipoprotein(a) phenotypes in patients with end-stage renal disease treated by hemodialysis or continous ambulatory peritoneal dialysis. J Am Soc Nephrol 1995;6:110-120.

[64] Milionis HJ, Elisaf MS, Tselepis A, Bairaktari E, Karabina SA, Siamopoulos KC. Apolipoprotein(a) phenotypes and lipoprotein(a) concentrations in patients with renal failure. Am J Kidney Dis 1999;33:1100-1106.

[65] Stenvinkel P, Heimburger O, Tuck CH, Berglund L. Apo(a)-isoform size, nutritional status and inflammatory markers in chronic renal failure. Kidney Int 1998;53:1336-1342.

[66] Zimmermann J, Herrlinger S, Pruy A, Metzger T, Wanner C. Inflammation enhances cardiovascular risk and mortality in hemodialysis patients. Kidney Int 1999;55:648-658.

[67] Kronenberg F, Lingenhel A, Lhotta K, et al. The apolipoprotein(a) size polymorphism is associated with nephrotic syndrome. Kidney Int 2004;65:606-612.

[68] Wanner C, Rader D, Bartens W, et al. Elevated plasma lipoprotein(a) in patients with the nephrotic syndrome. Ann Intern Med 1993;119:263-269.

[69] de Sain-van der Velden MG, Reijngoud DJ, Kaysen GA, et al. Evidence for increased synthesis of lipoprotein(a) in the nephrotic syndrome. J Am Soc Nephrol 1998;9:14741481.

[70] Kronenberg F, König P, Lhotta K, et al. Apolipoprotein(a) phenotype-associated decrease in lipoprotein(a) plasma concentrations after renal transplantation. Arterioscler Thromb 1994;14:1399-1404.

[71] Kronenberg F, Lhotta K, Konig P, Margreiter R, Dieplinger H, Utermann G. Apolipoprotein(a) isoform-specific changes of lipoprotein(a) after kidney transplantation. Eur J Hum Genet 2003;11:693-699. 
[72] Kerschdorfer L, Konig P, Neyer U, et al. Lipoprotein(a) plasma concentrations after renal transplantation: a prospective evaluation after 4 years of follow-up. Atherosclerosis 1999;144:381-391.

[73] Lindner A, Charra B, Sherrard DJ, Scribner BH. Accelerated atherosclerosis in prolonged maintenance hemodialysis. N Engl J Med 1974;290:697-701.

[74] Wilson PW, D'Agostino RB, Levy D, Belanger AM, Silbershatz H, Kannel WB. Prediction of coronary heart disease using risk factor categories. Circulation 1998;97:1837-1847.

[75] Cheung AK, Sarnak MJ, Yan G, et al. Atherosclerotic cardiovascular disease risks in chronic hemodialysis patients. Kidney Int 2000;58:353-362.

[76] Guz G, Nurhan Ozdemir F, Sezer S, et al. Effect of apolipoprotein E polymorphism on serum lipid, lipoproteins, and atherosclerosis in hemodialysis patients. Am J Kidney Dis 2000;36:826-836.

[77] Koch M, Kutkuhn B, Trenkwalder E, et al. Apolipoprotein B, fibrinogen, HDL cholesterol, and apolipoprotein(a) phenotypes predict coronary artery disease in hemodialysis patients. J Am Soc Nephrol 1997;8:1889-1898.

[78] Stack AG, Bloembergen WE. Prevalence and clinical correlates of coronary artery disease among new dialysis patients in the United States: a cross-sectional study. J Am Soc Nephrol 2001;12:1516-1523.

[79] Cressman MD, Heyka RJ, Paganini EP, O'Neil J, Skibinski CI, Hoff HF. Lipoprotein(a) is an independent risk factor for cardiovascular disease in hemodialysis patients. Circulation 1992;86:475-482.

[80] Degoulet P, Legrain M, Reach I, et al. Mortality risk factors in patients treated by chronic hemodialysis. Report of the Diaphane collaborative study. Nephron 1982;31:103-110.

[81] Hocher B, Ziebig R, Altermann C, et al. Different impact of biomarkers as mortality predictors among diabetic and nondiabetic patients undergoing hemodialysis. J Am Soc Nephrol 2003;14:2329-2337.

[82] Iseki K, Fukiyama K. Predictors of stroke in patients receiving chronic hemodialysis. Kidney Int 1996;50:1672-1675.

[83] Koda Y, Nishi S, Suzuki M, Hirasawa Y. Lipoprotein(a) is a predictor for cardiovascular mortality of hemodialysis patients. Kidney Int Suppl 1999;71:S251-S253.

[84] Kronenberg F, Neyer U, Lhotta K, et al. The low molecular weight apo(a) phenotype is an independent predictor for coronary artery disease in hemodialysis patients: a prospective follow-up. J Am Soc Nephrol 1999;10:1027-1036.

[85] Ohashi H, Oda H, Ohno M, Watanabe S, Sakata S. Lipoprotein(a) as a risk factor for coronary artery disease in hemodialysis patients. Kidney Int Suppl 1999;71:S242-S244.

[86] Schwaiger JP, Lamina C, Neyer U, et al. Carotid plaques and their predictive value for cardiovascular disease and all-cause mortality in hemodialysis patients considering renal transplantation: a decade follow-up. Am J Kidney Dis 2006;47:888-897.

[87] Shoji T, Emoto M, Shinohara K, et al. Diabetes mellitus, aortic stiffness, and cardiovascular mortality in end-stage renal disease. J Am Soc Nephrol 2001;12:21172124 . 
[88] Kalantar-Zadeh K, Kopple JD, Kilpatrick RD, et al. Association of morbid obesity and weight change over time with cardiovascular survival in hemodialysis population. Am J Kidney Dis 2005;46:489-500.

[89] Kalantar-Zadeh K, Kilpatrick RD, McAllister CJ, Greenland S, Kopple JD. Reverse epidemiology of hypertension and cardiovascular death in the hemodialysis population: the 58th annual fall conference and scientific sessions. Hypertension 2005;45:811-817.

[90] Nishizawa $Y$, Shoji T, Ishimura E, Inaba M, Morii H. Paradox of risk factors for cardiovascular mortality in uremia: is a higher cholesterol level better for atherosclerosis in uremia? Am J Kidney Dis 2001;38:S4-7.

[91] Kalantar-Zadeh K. What is so bad about reverse epidemiology anyway? Semin Dial 2007;20:593-601.

[92] Kalantar-Zadeh K, Block G, Humphreys MH, Kopple JD. Reverse epidemiology of cardiovascular risk factors in maintenance dialysis patients. Kidney Int 2003;63:793-808.

[93] Lowrie EG, Lew NL. Death risk in hemodialysis patients: the predictive value of commonly measured variables and an evaluation of death rate differences between facilities. Am J Kidney Dis 1990;15:458-482.

[94] Longenecker JC, Coresh J, Powe NR, et al. Traditional cardiovascular disease risk factors in dialysis patients compared with the general population: the CHOICE Study. J Am Soc Nephrol 2002;13:1918-1927.

[95] Longenecker JC, Klag MJ, Marcovina SM, et al. Small apolipoprotein(a) size predicts mortality in end-stage renal disease: The CHOICE study. Circulation 2002;106:28122818.

[96] Kronenberg F, Kathrein H, König P, et al. Apolipoprotein(a) phenotypes predict the risk for carotid atherosclerosis in patients with end-stage renal disease. Arterioscler Thromb 1994;14:1405-1411.

[97] Longenecker JC, Klag MJ, Marcovina SM, et al. High lipoprotein(a) levels and small apolipoprotein(a) size prospectively predict cardiovascular events in dialysis patients. J Am Soc Nephrol 2005;16:1794-1802.

[98] Kronenberg F, Ikewaki K, Schaefer JR, Konig P, Dieplinger H. Kinetic studies of atherogenic lipoproteins in hemodialysis patients: do they tell us more about their pathology? Semin Dial 2007;20:554-560.

[99] Pietzsch J, Lattke P, Julius U. Oxidation of apolipoprotein B-100 in circulating LDL is related to LDL residence time. In vivo insights from stable-isotope studies. Arterioscler Thromb Vasc Biol 2000;20:E63-67.

[100] Boaz M, Smetana S, Weinstein T, et al. Secondary prevention with antioxidants of cardiovascular disease in endstage renal disease (SPACE): randomised placebocontrolled trial. Lancet 2000;356:1213-1218.

[101] Tepel M, van der Giet M, Statz M, Jankowski J, Zidek W. The antioxidant acetylcysteine reduces cardiovascular events in patients with end-stage renal failure: a randomized, controlled trial. Circulation 2003;107:992-995. 
[102] Shoji T, Nishizawa $Y$, Kawagishi T, et al. Intermediate-density lipoprotein as an independent risk factor for aortic atherosclerosis in hemodialysis patients. J Am Soc Nephrol 1998;9:1277-1284.

[103] Vega GL, Grundy SM. Influence of lovastatin therapy on metabolism of low density lipoproteins in mixed hyperlipidaemia. J Intern Med 1991;230:341-350.

[104] Hörkkö S, Huttunen K, Korhonen T, Kesäniemi YA. Decreased clearance of lowdensity lipoprotein in patients with chronic renal failure. Kidney Int 1994;45:561-570.

[105] Hörkkö S, Huttunen K, Kesäniemi YA. Decreased clearance of low-density lipoprotein in uremic patients under dialysis treatment. Kidney Int 1995;47:1732-1740.

[106] Prinsen BH, Rabelink TJ, Romijn JA, et al. A broad-based metabolic approach to study VLDL apoB100 metabolism in patients with ESRD and patients treated with peritoneal dialysis. Kidney Int 2004;65:1064-1075.

[107] Chan PC, Persaud J, Varghese Z, Kingstone D, Baillod RA, Moorhead JF. Apolipoprotein B turnover in dialysis patients: its relationship to pathogenesis of hyperlipidemia. Clin Nephrol 1989;31:88-95.

[108] Ericsson S, Eriksson M, Vitols S, Einarsson K, Berglund L, Angelin B. Influence of age on the metabolism of plasma low density lipoproteins in healthy males. J Clin Invest 1991;87:591-596.

[109] Millar JS, Lichtenstein AH, Cuchel M, et al. Impact of age on the metabolism of VLDL, IDL, and LDL apolipoprotein B-100 in men. J Lipid Res 1995;36:1155-1167.

[110] Quaschning T, Koniger M, Kramer-Guth A, et al. Receptor-mediated lipoprotein uptake by human glomerular cells: comparison with skin fibroblasts and HepG2 cells. Nephrol Dial Transplant 1997;12:2528-2536.

[111] Pegoraro AA, Gudehithlu KP, Cabrera E, et al. Handling of low-density lipoprotein by the renal tubule: release of fragments due to incomplete degradation. J Lab Clin Med 2002;139:372-378.

[112] Kronenberg F, Trenkwalder E, Lingenhel A, et al. Renovascular arteriovenous differences in Lp[a] plasma concentrations suggest removal of Lp[a] from the renal circulation. J Lipid Res 1997;38:1755-1763.

[113] Kostner KM, Maurer G, Huber K, et al. Urinary excretion of apo(a) fragments. Role in apo(a) catabolism. Arterioscler Thromb Vasc Biol 1996;16:905-911.

[114] Mooser V, Seabra MC, Abedin M, Landschulz KT, Marcovina S, Hobbs HH. Apolipoprotein(a) kringle 4-containing fragments in human urine. Relationship to plasma levels of lipoprotein(a). J Clin Invest 1996;97:858-864.

[115] Kaysen GA, de Sain-van der Velden MG. New insights into lipid metabolism in the nephrotic syndrome. Kidney Int Suppl 1999;71:S18-21.

[116] Giordano M, De Feo P, Lucidi P, et al. Increased albumin and fibrinogen synthesis in hemodialysis patients with normal nutritional status. J Am Soc Nephrol 2001;12:349354.

[117] Kaysen GA. Albumin turnover in renal disease. Miner Electrolyte Metab 1998;24:55-63.

[118] Shoji T, Fukumoto M, Kimoto E, et al. Antibody to oxidized low-density lipoprotein and cardiovascular mortality in end-stage renal disease. Kidney Int 2002;62:2230-2237. 
[119] Randomised trial of cholesterol lowering in 4444 patients with coronary heart disease: the Scandinavian Simvastatin Survival Study (4S). Lancet 1994;344:1383-1389.

[120] Cannon CP, Braunwald E, McCabe CH, et al. Intensive versus moderate lipid lowering with statins after acute coronary syndromes. N Engl J Med 2004;350:1495-1504.

[121] Fried LF, Orchard TJ, Kasiske BL. Effect of lipid reduction on the progression of renal disease: a meta-analysis. Kidney Int 2001;59:260-269.

[122] Nishizawa Y, Shoji T, Tabata T, Inoue T, Morii H. Effects of lipid-lowering drugs on intermediate-density lipoprotein in uremic patients. Kidney Int Suppl 1999;71:S134-136.

[123] Seliger SL, Weiss NS, Gillen DL, et al. HMG-CoA reductase inhibitors are associated with reduced mortality in ESRD patients. Kidney Int 2002;61:297-304.

[124] Fellstrom BC, Jardine AG, Schmieder RE, et al. Rosuvastatin and cardiovascular events in patients undergoing hemodialysis. N Engl J Med 2009;360:1395-1407.

[125] Holdaas H, Holme I, Schmieder RE, et al. Rosuvastatin in diabetic hemodialysis patients. J Am Soc Nephrol 2011;22:1335-1341.

[126] Wanner C, Krane V, Marz W, et al. Atorvastatin in patients with type 2 diabetes mellitus undergoing hemodialysis. N Engl J Med 2005;353:238-248.

[127] Chan KE, Thadhani R, Lazarus JM, Hakim RM. Modeling the 4D Study: statins and cardiovascular outcomes in long-term hemodialysis patients with diabetes. Clin J Am Soc Nephrol 2010;5:856-866.

[128] Krane V, Wanner C. Statins, inflammation and kidney disease. Nat Rev Nephrol 2011;7:385-397.

[129] Baigent C, Landray MJ, Reith C, et al. The effects of lowering LDL cholesterol with simvastatin plus ezetimibe in patients with chronic kidney disease (Study of Heart and Renal Protection): a randomised placebo-controlled trial. Lancet 2011;377:2181-2192.

[130] Epstein M, Vaziri ND. Statins in the management of dyslipidemia associated with chronic kidney disease. Nat Rev Nephrol 2012;8:214-223. 